\title{
Multimodal Analysis of Innisfree's Brand History
}

\author{
Vanessa Velancia Prajogo, Setefanus Suprajitno \\ English Department, Faculty of Languages and Literature, Petra Christian University, Siwalankerto 121- 131, \\ Surabaya 60236, INDONESIA \\ E-mail: m11416007@john.petra.ac.id, steph@ petra.ac.id
}

\begin{abstract}
Fierce competition in increasing market share growth prompts companies to strive to create a memorable brand identity. One common method adopted by some companies is using a video advertisement, which contains the message they want to convey about their product or brand. This message is intended to persuade people who see the advertisement to buy their product. In doing so, companies usually use the visual, linguistic, spatial and other semiotic resources, often referred to as a semiotic mode. Through the case study of Innisfree's video advertisement "Brand History," we analyze how Innisfree uses linguistic, spatial, and visual mode for creating a message that enables consumers to remember and to persuade them buy its product. Our findings show that the message in the video advertisement is that Innisfree is a beauty product that upholds nature as its ingredients, has high quality, and preserves the environment where it takes its ingredients.
\end{abstract}

Key Words: Advertisement, Persuasion, Modes, Verbal and Non-verbal expressions.

\section{INTRODUCTION}

Nowadays, there are a lot of big and small companies existing in this world. Each has its own brand name in order to distinguish itself from others, with the purpose of people knowing its brand. Since there are many brands in the world, every company need to think of methods to make its brand or products stand out in the market so that consumers remember the brand or the products. One convenient way of doing so is employing advertisements.

Advertisements are usually intended to persuade potential consumers to buy the products advertised by stating their benefits. However, development in advertising communication changes the way the persuasion is made. At present many advertisements use video that has storylines for introducing the product or the brand advertised to potential consumers, which make the advertisements more interesting. This kind of video is popularly known as a signature story. A signature story combines narration and message that have been put together in such a way that it provides an information that companies want to convey to their potential consumers through their brand in an interesting way. Many companies often include the history of their development such as their personal story or an honest information to increase the credibility of their business and the products that they sell. This method is proven to be excellent in building a positive brand image (Pfeuffer, 2015). Innisfree, a well-known cosmetic company in Korea, is an example of a company that uses this method. It uses a signature story to introduce its brand in the U.S for the first time through its official Youtube channel two years ago.

In its signature story, which introduced its brand for the first time in the U.S., Innisfree used a historical concept to illustrate how it was founded. It used this historical theme to describe how its founder was inspired by his mother in making natural skincare that came from nature. With such a concept that uncovers the history of its brand, Innisfree presents personal stories that build their 'natural' brand image, that is, the ingredients used for its products are natural ingredients that are taken from Jeju Island (Innisfree, 2018). The idea of natural brand image is also reinforced through highlighting Jeju Island, recognized globally as a UNESCO World Natural Heritage site, as the place where Innisfree acquires its ingredients for its product. In this way, Innisfree tries to increase people's trust in trusting components and ingredients originating from Jeju island (Melvioni \& Suprajitno, 2017). Through this historically-themed signature story advertisement, Innisfree's video becomes an intermediary to expose the audience to the semiotic world where the audience actively interprets the meaning of the video itself so that the process is engaging and convincing. 
In this paper, we are interested in analyzing Innisfree's video advertisement, entitled "Brand History," posted on official Innisfree USA YouTube account, which can be found at https://www.youtube.com/watch?v=0mTpuKMqUgQ. This video is chosen because it is the only promotional video having a storyline that is not divided into several parts, and it uses a lot of semiotic signs for promoting the items of Innisfree's products. Since this video uses semiotic resources in various modes, we use the theory of Multimodal Discourse Analysis that is adapted by Chan \& Chia (2017) \& Anstey and Bull (quoted from Kress and van Leeuwen, 2006). This theory basically acts as an instrument on how all the elements in the video will support each other and help the meaningmaking process so that when the elements are combined in the right way, it will help the company to share their information that they want to convey to their consumer and reduce the possibility of miscommunication that will occur.

In our analysis, we categorize the expression of message in this this video into three general modes, namely, linguistic, spatial, and visual mode. The linguistic mode is the narrative shown in the video represented by letter, word, and sentences. Spatial mode refers to the space or background in every scene involving angles when the video is taken. Visual mode refers to the moving images such as objects and places, involving colors, lighting, shapes etc. We decide to exclude other modes because the other modes, namely audio, gestural and, oral modes are not so prominent in this video. These three semiotic modes are analyzed, and then the relations of these modes are examined in order to uncover the element of persuasion. Thus, in this paper, we want to analyze how the message in the advertisement video is conveyed through linguistic, spatial, and visual modes, and how the semiotic modes (linguistic, spatial, and visual) are related to each other in order to strengthen the message and to show the power of persuasion in the video.

We use the theory of Multimodal Discourse Analysis theory because, firstly, multimodal discourse analysis has an important role in providing information and convincing the audience to buy the goods without actually realizing that the advertisement is actually a persuasion device. Secondly, verbal expression, in this case, the text enclosed in an advertisement, actually contains many hidden meanings, for example, the use of metaphors or synonyms to form an expression so that later when verbal and non-verbal expressions are combined into one, it will form an identity or message that the company wants to convey to their viewers (Kress \& van Leeuwen, 2006).

\section{METHOD}

In the analysis, we use a descriptive qualitative research method formulated by Schreier (2012). This method is chosen because of the need for a detailed explanation of each mode and its connection to people's interpretation regarding the power of persuasion. In addition, a qualitative research is more suitable than quantitative research because explanations are needed to clarify a problem in more detailed and deep way to develop a hypothesis.

Data for this paper are taken from Innisfree's promotional video entitled "Brand History." The video can be found in official Innisfree USA YouTube account. The duration of the video is approximately 2.36 minutes with the link of the video. The forms of the data are semiotic resources, which we categorize into three modes, namely, linguistic, spatial, and visual modes (Chan \& Chia, 2017; Anstey \& Bull, quoted in Kress \& van Leeuwen, 2010). Linguistic modes focus more on the vocabulary, metaphor, and structure of the text presented in the video. Spatial modes is concentrated more on the direction and position in space such as how the object is placed in the video whether in the foreground or at the background of the video. Meanwhile visual modes will discuss colors, perspective, shapes, gaze, and viewpoints.

\section{FINDINGS AND DISCUSSION}

Through multimodal discourse analysis on Innisfree's commercial video "Brand History," we would like to find out how semiotic modes such as the linguistic, spatial, and visual modes are used in the process of creating a meaning in this video, and how these modes support each other in order to 
strengthen the message and to create a persuasion effect. Our findings show that the message in the video advertisement is that Innisfree is a beauty product that upholds nature as its ingredients, has high quality, and preserves the environment where it takes its ingredients.

\section{Linguistic Mode}

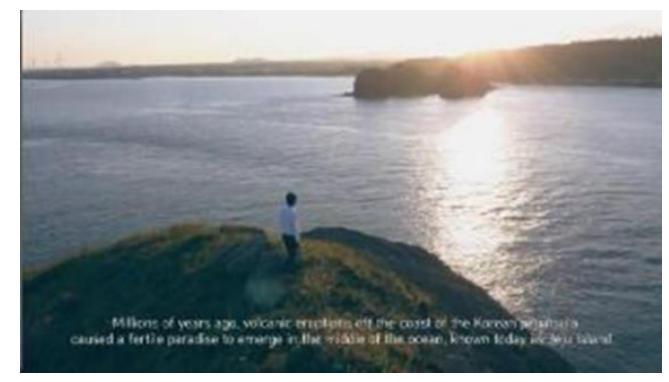

Figure 1

Text: Millions of years ago, volcanic eruptions off the coast of Korean peninsula caused a fertile paradise to emerge in the middle of the ocean, known today as Jeju Island.

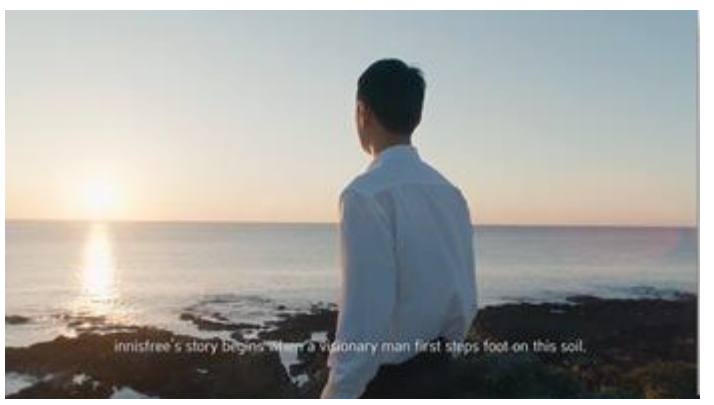

Figure 2

Text: Innisfree's story begins when a visionary man first steps foot on this soil.

Linguistic mode refers to written or the spoken text, whether it is on the printed media or said by someone else. The text usually becomes the main medium to convey the message. The text involves elements such as vocabulary and grammar. Word choice is also an important element in conveying something especially in advertising. In the video of Innisfree's advertisement, the linguistic modes are presented through the narration in a form of text which is located at the bottom center in the video, as seen in Figure 1 and 2 above.

In analyzing the linguistic mode, as exemplified in Figure 1, we take a look at the narration that says "Millions of years ago, volcanic eruptions off the coast of the Korean peninsula caused a fertile paradise to emerge in the middle of the ocean, known today as Jeju Island." The beginning of the video begins with the phrase "Millions of years ago." This phrase invites the audience to imagine the situation where the event took place a long time ago. The structure of the sentence uses simple past tense to show that Jeju Island has been existing since time immemorial. With this kind of structure, the audience is also invited to visualize as if they are reading a story which is adapted from a true story. The word "fertile" is used to describe the condition of the soil resulting from the eruption of a mountain which is rich in nutrients. Soil that developed from former volcanic activity is fertile soil, which is ideal for growing vegetation because it is rich in minerals. In addition, because Jeju Iisland is an isolated area until Innisfree's inventors come and cultivate various plants there, the mineral contained in plants is still pure and has not been touched by humans. Thus, the linguistic mode here implies that the products produced are rich in minerals and good for skin vitality.

The narration in Figure 2 uses the word "soil," instead of using the word "place." According to Merriam-Webster Dictionary 2020, the word "soil" refers to the most top layer of earth where tress and plant grows. In the context of Figure 2, the word "soil" has a connection with the state of the vast land where, the man standing there, Sunghwan Suh, sees the condition of Jeju Island that has not been used to cultivate plants. In the narration, Sunghwan Suh's name is not written as it is and replaced by the word "visionary man" to introduce him for the first time. The word visionary has the meaning of someone with the ability to plan the future with his or her intelligence and competency. These two words are related to each other since Sunghwan Suh, the founder of Innisfree, sees that the quality of soil, water, and the air of Jeju Island is very pristine. But unfortunately, this cannot be put to good use by the people who live there. So, it can be said that in the linguistic mode, the "visionary man" in the sentence refers to his vision to cultivate something on the island, utilize the natural resources there and also preserve it, as reflected in the vision and mission of Innisfree. 
Prajogo, Suprajitno: Multimodal Analysis of Innisfree's Brand History

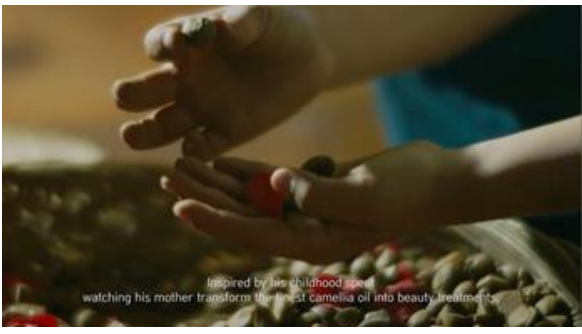

Figure 3

Text: Inspired by his childhood spent watching his mother transforms the finest camellia oil into beauty treatment.

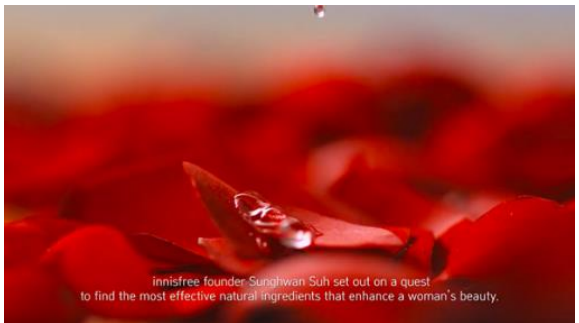

Figure 4

Text: Innisfree founder Sunghwan Suh set out on a quest to find the most effective natural Ingredients that enhance a woman's beauty.

The narration in the linguistic mode in Figure 3 is "Inspired by his childhood spent watching his mother transforms the finest Camelia oil into beauty treatment". This narration uses past tense, which as if brings the audience to the narrative past. This text shows Sunghwan Suh was inspired by what his mother did in making skincare products. Besides that, the word "finest" meant that his mother was not haphazardly choosing ingredients which were going to be used as skincare products. Instead, she chose to use high-quality ingredients. The word "finest," according to the MerriamWebster Dictionary, comes from the word "fine," which means it can be well received and quite good. The word "finest" is the superlative form, which in this context expresses the idea that the mother uses the best out of best ingredients. Another thing that is worth mentioning is the word "spent" which follows the word "childhood." It implies that Sunghwan Suh observed how his mother made beauty products, for example, she often processed Camellia seeds into Camellia oil, one ingredient for beauty products. It means that her mother spent much of her time for making the products, and he spent his childhood time helping his mother and learning how to make beauty products. Eventually, he became interested and wanted to continue his mother's effort. As seen in Figure 4, the linguistic mode employs the word "quest," a word less commonly used for explaining his efforts in finding the best ingredients that can enhance women's beauty. According to Merriam-Webster dictionary, "quest" has the meaning of a long search especially for achieving perfection. This linguistic mode implies that without realizing it, seeing his mother spend her time experimenting with traditional Korean ingredients such as green tea, camellia seeds, and ginseng, he developed a passion to find the perfect ingredients to make women beautiful. Thus, the linguistic mode aims to show the quality of the ingredients used to make the beauty product is a superior one.

\section{Spatial Mode}

Spatial modes include the direction and position in space to create a point of view, for example, worm's-eye view, which is taken from below that the view of people taken seems imposing. The background of the scene which is usually the location of the scene. The camera angle and camera position to show the overall perspective of the scene. Figure 1, for example, shows that the setting of the video is taken in a secluded island which can be assumed as Jeju Island. The way the video is taken from afar, known as bird's-eye view, gives the audience have a better angle of the view of the island, which is surrounded by waters and which does not show any signs of housing residents, transportation, factories and others. This creates and impression of how pristine the island is. The spatial mode in this frame aims to show that Jeju Island is a pristine island. It implies that the material produced and used to make beauty products is also natural.

In Figure 2, the setting of the place where the video is taken is located on the seashore, which can be assumed as the seashore of Jeju Island. The portrait of Sunghwan Suh is taken through the medium shot method which shows half of the actor's body turning his back on the audience and giving a mysterious impression especially to the viewers of Innisfree USA YouTube channel, many of whom do not know who Sunghwan Suh is. The background in this figure is the sea and the coast with centered composition technique to show the balance of the picture. The use of beach settings aims to 
emphasize the beauty of nature by displaying the color of nature and this gives the impression that the cosmetics are a beauty product which are natural and safe to use by humans.

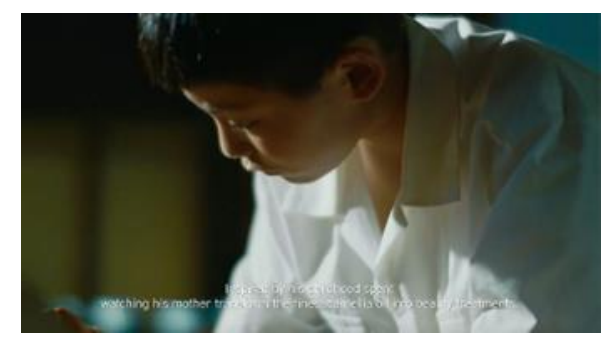

Figure 5



Figure 7

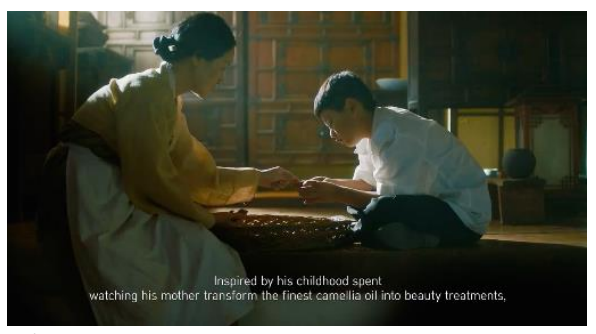

Figure 6



Figure 8

The spatial mode in Figure 5 and 6 shows that the scene is taken at Sunghwan's house where he usually sees and helps his mother for hours to process Camellia seeds into Camellia oil. The background of the place in which this scene is taken becomes an important background where he got his inspiration and interest in utilizing traditional Korean ingredients, such as green tea, ginseng, and Camellia seed oil to get their benefits for health and beauty. Here, what Innisfree is trying to convey by inserting the background of Sunghwan Suh's house is to show the historical value representing the "tradition" that he got from his mother in making beauty product treatments based on natural ingredients. Tradition refers to something that is handed down through generations through habits. Thus, the scene in these figures show the value of their family tradition is to make everything from natural ingredients. The medium close-up shots in Figure 7 and 8 renders the scene emphasizing on the ingredients that they use and recording the process from the beginning. The process includes how they choose raw materials with their own hands to sort out which seeds that have good quality until grinding those ingredients in order to produce Camellia seed oil. The camera positions in Figure 7 and 8 give the access to see the process on how they choose the ingredients carefully. In order to capture the moment when Sunghwan Suh is helping and learning from his mom, centered composition captures two people in the frame without making one person blocking another which makes the picture look balanced (Figure 6). The spatial mode in the figures above wants to convey the value of the "tradition" that is passed through generations in making beauty products from handpicked ingredients and traditionally processed.

The salience that is quite noticeable when Figure 6 starts to show, the light is slightly dimmer and the color of the video slightly changes from bluish into brownish color to indicate the time difference that would differentiate the present and the past. Another thing that is prominent in the visual mode in Figure 5-8 is that the raw ingredient will be processed into the final ingredients by using a traditional method. The way they process all raw materials in order to become the final product is a difficult process which requires time and patience to get high-quality products. In addition, the use of pictures where they are making oil from camellia seeds with traditional tools show that Innisfree was born from a tradition which was taught by the founder's mother when he was a child, when he had to make Camellia oil directly from its seeds. Figure 5 and 6 show Sunghwan Suh's expression, that is, he paid attention to all the information given by his mother to make beauty products. As seen in this scene, making beauty products is something that her mother is doing seriously where she can only use a few Camellia seeds from many seeds to get good oil. Thus, from 
the sentence above it can be concluded that the ingredients used to make beauty products are obtained from high quality ingredients that are selected by maintaining their natural conditions.

\section{Visual Modes}

Visual modes focus on things that are "stand out" including color, shape, and viewpoint as well as the meaning of the color and the cultural background if there is one. As Paltridge (2012) states, meaning is not only obtained from the use of words, but also from other modalities, such as colors.

In Figure 1 and 2, the visual modes depict the color of nature by using the green and blue colors. These colors not only emphasize nature, but also give the healing power for human eyes and represent the base color of the overall video. The color depicted in the video is not at the maximum level but in fact quite low, in line with the sun that has not come out yet in order to give the visual of fresh morning air. The collaboration between the lighting that is not too bright and the green and blue colors illustrates the purity of nature that people often look for in a perfect way. In other words, the visual modes depict the lighting and the green and blue colors as the highlight of this scene. They emphasize not only nature, but also the time of the day, which is still early in the morning. This can be seen through the location of the sun that is still close to the horizon. The dim light also supports the representation of the gentle morning light as the sun rises which is a sign for people to start their activities. So, in this video, gentle sunlight when the sun begins to shine implies the "beginning" of the story of Innisfree.

The visual depiction of the zoomed beauty ingredients, as seen in Figure 3 and 4, makes the texture of the Camellias and its oil clearly visible. Figure 8 shows that the oil extract is very clear and the camellia flower looks very fresh. The vibrant reddish color of the flower also gives an impression that the ingredients and the results of the beauty products are taken from selected ingredients that have good quality and are processed in a good way.

Innisfree claims itself as a company that implements fair trade principle, green initiatives, and preservation efforts. Innisfree seeks to prove and distinguish itself from other competitors by showing its commitment to preserving nature and human resources that are rarely owned by companies in general. The first thing is about "fair trade policies" for farmers which are often forgotten by companies and often make farmers get unfair treatment in terms of payment systems, equality between farmers and producers and transparency. By implementing a fair-trade system, Innisfree wants to show that they are a company that upholds humanities by creating transparent trading where the relationship between producers and farmers is not just an economic relationship. Instead, it establishes a relationship based on trust. The visual modes in Figure 9 show a farmer who is choosing Camellias and Bija that are going to be used as a beauty product. She represents the farmers who work for Innisfree in Jeju Island to which Innisfree contributes economically by investing in the island and providing employment to the people and the farming community.

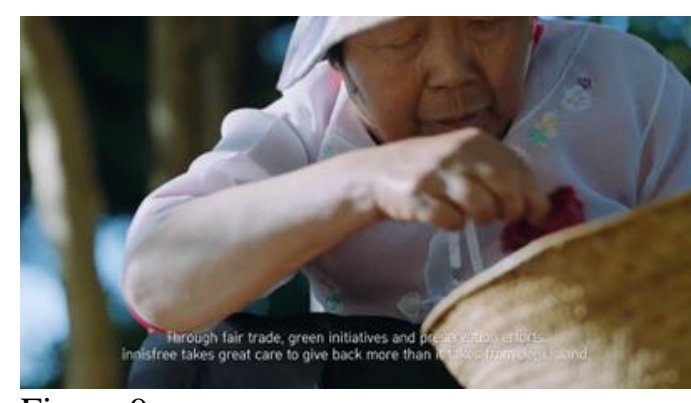

Figure 9



Figure 10

Innisfree's green initiatives and preservation efforts are depicted in the scene whose visual modes depicts Innisfree's customers put the empty bottles of the product in the place that Innisfree 
has provided at its outlets. Innisfree provides a recycling bin to encourage its customers to implement recycling practices in which plastic is one of the largest waste contributors that are difficult to recycle and one of the biggest enemies of nature. Here, Innisfree proves and actualizes its vision with real action and providing facilities for their consumers to partake in the program. Thus, Innisfree indirectly proves the efforts of their company in proving that they care and remain responsible for nature even if they extract their cosmetic ingredients from it.

\section{Interrelation of Each Mode and Persuasion Points}

In the video discussed here, Innisfree uses the linguistic, spatial, and visual modes to create a message that it wants to convey to its audience. The interrelation of these three modes support them in strengthening the message, and creating a persuasion effect. Here we select several scenes the interrelation of the modes can be seen most clearly. The first set of images that we take are shown in Figure 3, 4, 5, 6, 7, and 8. These figures show little Sunghwan and his mother in the middle of selecting the camellia seeds, the main ingredients for making camellia oil. From the three modes that we analyze, we can see their connections on how they support each other to deliver messages.

The text in the scene wants to convey a meaning that Sunghwan Suh got his inspiration to make beauty treatments only from high-quality and natural ingredients from his mother. This message is supported by the visual mode in one particular scene. It shows that Sunghwan Suh and his mother sorting Camellia seeds to ensure that they only use finest quality Camellia seeds as ingredients to make oil amongst the Camellia seeds they have harvested. The word "childhood" in the narration is also supported by the visualization of Sunghwan Suh, who is still a child, with his mother spending their time together to process Camellia oil. The linguistic mode supports the spatial mode in that scene, in which the medium close-up shot technique is used to capture Sunghwan Suh's and his mother's activity, processing Camelia seeds into Camellia oil, using the word "transforms" in the video. Through the scenes above, the persuasion point which Innisfree wants to inform to its potential customer is that Innisfree comes from a tradition that upholds the quality of the ingredients. Therefore, it will make the customer convinced in choosing Innisfree's product.

The second set of images (Figure 9 and 10) shows a local farmer, and then some people in a store putting their used bottles in a container. The interrelation of linguistic mode and the visual mode can be seen from all the pictures above. When the narration "Through fair trade, green initiatives and preservation efforts, Innisfree takes great care to give back more than it takes from Jeju Island" appears in the video, the text appears at the same time with the picture of the farmers, people putting their empty bottle and the recycle bin. Then, the second interrelation of all the modes can be seen when the spatial mode simultaneously supports the visual and linguistic mode to elaborate the "preservation effort". The visual mode is supported by the close up shot of the people putting their bottle to the recycle bin and the linguistic mode is supported by the spatial mode when the word recycle is zoomed in to make the audience know exactly how Innisfree will preserve the nature which is by turning the plastic into another item through recycling process.

The persuasion point is depicted through the word fair-trade and the preservation effort. It indicates that Instead of getting one benefits, when they buy Innisfree products, people will get two benefits. First by buying the product, it will ensure that the farmers will receive decent payment and treatment because of the fair-trade policy and people will also feel happy that they can protects the environment through the recycling program when at the same time they can enhance their beauty. This will make people happy because Innisfree products provide benefits not only for them, but for others as well.

After analyzing the interrelation of all of the modes, it occurs to us that each mode supports each other to achieve a greater message. The combination of all these modes is not only useful for conveying clearer information but also has a persuasive effect on the video advertisement. Hence, all the modes presented in the video advertisement work together to make a message that will have an impact that leads to persuasion. 
Prajogo, Suprajitno: Multimodal Analysis of Innisfree's Brand History

\section{CONCLUSION}

In this paper, through the study of an Innisfree promotional video, we find that each mode has its respective role and the combination of all of them will create a more meaningful message so that the message can be transmitted efficiently. Obviously, the verbal expression in the video advertisement, that is, the narration, is supported by the non-verbal expression. Both of them are consistent in delivering the message to the audience throughout the video advertisement. Multimodal Discourse Analysis theory helps us analyze how Innisfree uses the linguistic, spatial, and visual mode in voicing out and strengthening its message and persuasion power.

The video that we study is an advertisement taken from Innisfree USA official YouTube channel. The video, which entitles "Brand History," contains many semiotic resources, such as linguistic, spatial, and visual mode, which helps Innisfree convey its message as a high-quality natural beauty product. The linguistic mode in this video advertisement acts as the main instrument for delivering the information in the narrative form. This mode conveys information in the form of reality or facts, which makes this mode have the highest importance since the story in this advertisement is mostly conveyed through the text. The visual and the spatial modes serve to provide what the linguistic mode cannot provide such as images or visualizations to support the information it displays. For example, visual mode displays the movements of an object or individuals and spatial mode displays how the position of the objects is arranged in the video. Therefore, all these combinations of modes will later create a bigger meaning that leads to a persuasion effect.

In the end, Innisfree's video advertisement is formed and shaped by non-verbal and verbal elements, such as the visual, spatial, and linguistic modes. The linguistic mode has been selected meticulously in order to be able to deliver the intended message. It is supported by the spatial and visual mode so that the advertisement works efficiently. The advertisement uses a historical-themed storyline, and this is unique because the advertisement does not promote the product directly. Instead, it uses a story-line to communicate its message and to persuade people to buy its products. By analyzing its advertisements, we find the message that Innisfree wants to deliver to its customers and audience, that is, Innisfree is a beauty product brand that upholds nature as its ingredients, has high quality, and at the same time preserves the environment where it takes its ingredient. These messages are depicted throughout the video advertisement by exposing the history on how Innisfree was first founded, the process of the product making until to the point Innisfree's campaign to preserve the environment as its way to honoring nature.

\section{REFERENCES}

Chan, A. \& Chia, C. (2017). Re-defining 'reading' in the 21 st century: accessing multimodal texts. Beyond Words, 5(2), 98-1.

Innisfree (2018). Brand concept. Retrieved from http://www.innisfree.com/id/id/BrandPage.do?pageName=brand_concept.

Kress, G., \& van Leeuwen, T. (2006). Reading images. New York: Routledge.

Melvioni, K., \& Suprajitno, S. (2017). Innisfree's Brand Image through Jeju Island Campaigns. K@ta Kita, 5(1), 95-99. doi: 10.9744/katakita.5.1.95-99.

Paltride, B. (2012). Discourse analysis: An introduction. $2^{\text {nd }}$ Ed. London: Bloomsbury.

Pfeuffer, A. (2015). The Effectiveness of Radical Openness in Online Advertising. Communication Research Reports, 32(1), 45-53. doi: 10.1080/08824096.2014.989974.

Schreier, M. (2012). Qualitative content analysis in practice. Thousand Oaks: Sage Publications. 